\title{
Immune thrombocytopenia induces autophagy and suppresses apoptosis in megakaryocytes
}

\author{
ZHANSHU LIU $^{1}$ and TONGHUA MEI ${ }^{2}$ \\ ${ }^{1}$ Department of Hematology, Yongchuan Hospital of Chongqing Medical University; \\ ${ }^{2}$ Department of Respiratory and Critical Care Medicine, The First Affiliated Hospital of \\ Chongqing Medical University, Chongqing 400016, P.R. China
}

Received January 4, 2018; Accepted May 5, 2018

DOI: $10.3892 / \mathrm{mmr} .2018 .9373$

\begin{abstract}
Immune thrombocytopenia (ITP) is the main pathogenesis of excessive platelet destruction and abnormal megakaryocyte apoptosis, however, the mechanism underlying this abnormality in megakaryocytes remains to be elucidated. Since autophagy and apoptosis are closely interrelated, it can be speculated that the abnormal apoptosis of ITP megakaryocytes is associated with autophagy. To test this hypothesis, a total of 14 patients with ITP and 23 healthy controls were recruited. MEG-01 cell line was cultured in vitro, and morphological changes were observed by light microscopy, apoptosis was evaluated by flow cytometric analysis of Annexin V-FITC/propidium iodide staining and western blot analysis of B-cell lymphoma (Bcl)-2, Bcl-associated $\mathrm{X}$ protein (Bax), Beclin-1 and cleaved caspase 3. Apoptotic abnormalities and autophagy were observed in the ITP plasma group. Furthermore, Bax expression was downregulated, while Beclin-1 was upregulated. Chloroquine can block autophagy induced by ITP and remove the ITP plasma inhibition of apoptosis. Therefore, it may be concluded that ITP may induce autophagy, the inhibition of which may be a novel treatment for ITP.
\end{abstract}

\section{Introduction}

Immune thrombocytopenia (ITP) is an acquired autoimmune disorder, which is characterized by excessive bleeding (1). Although ITP is classified as a benign disease, the quality of life of patients with ITP is lower than that of patients with cancer. A comprehensive understanding of the pathogenesis of ITP is a means of improving the quality of life of these patients. To date,

Correspondence to: Dr Tonghua Mei, Department of Respiratory and Critical Care Medicine, The First Affiliated Hospital of Chongqing Medical University, 1 Gang Yuan Friendship Road, Chongqing 400016, P.R. China

E-mail: mtonghua@163.com

Key words: immune thrombocytopenia plasma, autophagy, apoptosis, megakaryocyte research on the pathogenesis of ITP has been focused mainly on platelets or megakaryocyte apoptosis, which are destroyed or functionally inhibited by humoral and cellular immunity $(2,3)$, with the latter receiving far less attention than the former. In ITP patients, megakaryocytes predominantly remain at the granule megakaryocyte stage, although the fate of these cells is unclear. Programmed cell death can be divided into several categories, including type I (apoptosis) and type II (autophagic death) (4). Houwerzijl et al (5) demonstrated increased vacuoles, plasma membrane thickening and chromatin condensation in the mitochondria and endoplasmic reticulum (ER) of ITP megakaryocytes. This may result in endoplasmic reticulum stress, leading to autophagy $(6,7)$. Autophagy is a biological process, where cytoplasmic macromolecules self-degrade through the lysosomal pathway (8). Chloroquine (CQ) can affect the $\mathrm{pH}$ of lysosomes and the ability of lysosomes to degrade proteins, as well as block the fusion of autophagosomes and lysosomes, which subsequently inhibits autophagy (9). In recent years, great progress has been made in the study of the association between autophagy and tumors, however, the role of autophagy in hematological diseases is not yet clear. Previous studies have demonstrated that premature death of megakaryocytes may induce ITP thrombocytopenia and megakaryocytes undergo autophagic (10). Therefore, it was hypothesized that megakaryocytes are likely to undergo autophagy in ITP patients. In the present study, the effect of autophagy on megakaryocytes was investigated to identify the mechanism of action in ITP using the human megakaryoblast leukemic cell line MEG-01 and plasma obtained from patients with ITP.

\section{Materials and methods}

Cell culture. The human megakaryoblast leukemic cell line MEG-01 was obtained from Guangzhou JENNIO Biological Technology (Guangzhou, China). Cells were maintained in RPMI-1640 media (Gibco; Thermo Fisher Scientific, Inc., Waltham, MA, USA) supplemented with $10 \%$ heat-inactivated fetal bovine serum (Gibco; Thermo Fisher Scientific, Inc.) and $1 \%$ penicillin/streptomycin, and cultured in an incubator at $37^{\circ} \mathrm{C}$ under $5 \% \mathrm{CO}_{2}$ and $95 \%$ humidity.

Patient information. A total of 14 patients [11 females and 3 males, median age 49 years (range 23-68 years)] 
with ITP and 23 healthy controls [16 females and 7 males, median age 46 years (range 22-66 years)] were recruited. All patients were newly diagnosed and excluded from other autoimmune diseases and malignant tumors. Venous blood samples $(2 \mathrm{ml})$ were collected into sterilized plastic tubes prior to any drug treatment by the Laboratory of Hematology (Yongchuan Hospital of Chongqing Medical University, Chongqing, China) between June and December 2016. Plasma was separated by centrifugation at room temperature $(2,000 \times \mathrm{g}$ for $10 \mathrm{~min})$, and aliquots were frozen at $-80^{\circ} \mathrm{C}$ for subsequent experiments. The present study was approved by the Research Ethics Board of the Yongchuan Hospital of Chongqing Medical University and informed consent was obtained from all participants.

Cell processing method. i) No plasma group: Meg-01 cells were cultured in plasma-free 1640 medium. ii) Normal plasma group: Meg-01 cells were co-cultured with normal control plasma. iii) ITP plasma group: Meg-01 cells were co-cultured with ITP patient plasma. Each experiment was repeated three times.

Morphological changes. i) Meg-01 cells were taken in the logarithmic growth phase, centrifuged at $100 \mathrm{x} g$ for $5 \mathrm{~min}$ at room temperature, and an appropriate amount of serum-free 1640 culture solution was added, mixed. The cell density was adjusted to $1 \times 10^{5} / \mathrm{ml}$, to inoculate a six-well plate. Each group was inoculated with 2 wells and $1.8 \mathrm{ml}$ cell suspension was added to each well. ii) According to the experimental group, $200 \mu 1$ serum-free medium, $200 \mu 1$ normal control plasma, and $200 \mu 1$ ITP plasma were added to each well for incubation for $36 \mathrm{~h}$ at $37^{\circ} \mathrm{C}$ in a humidified cell incubator containing $5 \% \mathrm{CO}_{2}$. iii) After 36 h, $2 \mathrm{ml}$ cell suspension was taken from each group and transferred to two $1.5 \mathrm{ml}$ EP tubes and centrifuged at $100 \mathrm{x} \mathrm{g}$ for $5 \mathrm{~min}$, at room temperature. iv) The cells were washed twice with PBS (at $400 \times \mathrm{g}$ for $5 \mathrm{~min}$, at room temperature and the cells were harvested to a cell concentration of $1 \times 10^{5} / \mathrm{ml}$. v) The different groups of cells were placed on labelled polylysine slides. vi) Slices were placed in a ventilated room and allowed to dry naturally. Then the slide was placed on the staining rack. vii) The remaining steps are the same as in part i. viii) The slides were observed under oil by laser scanning confocal microscope.

Evaluation of apoptosis by flow cytometric analysis of Annexin V-FITC/propidium iodide (PI) staining. To investigate cell apoptosis, MEG-01 cells $\left(2 \times 10^{5}\right.$ cells $\left./ \mathrm{ml}\right)$ were obtained post-treatment according to the flow cytometry kit protocol. Following this, cells were stained with Annexin V/PI (Beyotime Institute of Biotechnology, Haimen, China), according to the manufacturer's protocol and then analyzed with a FACStar PLUS ${ }^{\mathrm{TM}}$ (BD Biosciences, Franklin Lakes, NJ, USA).

Evaluation of cell autophagy by Lyso-Tracker Red/ansylcadaverine (MDC) assay and flow cytometry. Cell death of MEG-01 cells $\left(2 \times 10^{5}\right.$ cells $\left./ \mathrm{ml}\right)$ was investigated. Autophagosomes were marked with Lyso-Tracker Red (Beyotime Institute of Biotechnology, Shanghai, China) at $37^{\circ} \mathrm{C}$ for $30 \mathrm{~min}$ and MDC (Beijing Solarbio Science and Technology Co., Ltd., Beijing, China) at room temperature for $30 \mathrm{~min}$ in the dark, according to the manufacturer's protocol. Autophagy was detected under a fluorescence microscope using flow cytometric analysis as described previously (11).

Western blot analysis. Following washing of the treated cells three times using PBS, MEG-01 cells were harvested and lysed in RIPA buffer (Sigma-Aldrich; Merck KGaA (Darmstadt, Germany) containing $0.1 \mathrm{mg} / \mathrm{ml}$ of phenylmethane sulfonyl fluoride on ice for $30 \mathrm{~min}$. Supernatants were subsequently isolated via centrifugation at $12,000 \mathrm{x}$ g for $5 \mathrm{~min}$ at $4^{\circ} \mathrm{C}$, Protein content was determined by BCA. Following this, equal amounts of protein from cell lysates $(30 \mu \mathrm{g}$ total protein per lane) were separated via 10\% SDS-PAGE and then transferred to polyvinylidene difluoride membranes. Following this, membranes were blocked for $1 \mathrm{~h}$ at room temperature with 5\% skim milk powder in Tris-buffered saline containing $0.1 \%$ Tween-20 (TBST). Western blot analysis was performed according to standard protocol using the following primary antibodies at room temperature for $2 \mathrm{~h}$ : Anti-B-cell lymphoma (Bcl)-2 mouse monoclonal antibody (cat. no. 15071; 1:1,000; CST Biological Reagents Co., Ltd., Shanghai, China) and anti-Bcl-associated X protein (Bax) rabbit polyclonal antibody (cat. no. 2774; 1:1,000; CST Biological Reagents Co., Ltd.); anti-Beclin-1 rabbit polyclonal antibody (cat. no. ab207612; 1:1,000) and anti- active Caspase-3 rabbit monoclonal antibody (cat. no. ab2302; 1:1,000; both Abcam, Cambridge, UK), and anti- $\beta$-actin mouse monoclonal antibody (cat. no. AA128; 1:1,000; Beyotime Institute of Biotechnology, Shanghai, China). Membranes were subsequently washed three times for $10 \mathrm{~min}$ each with TBST; incubated with horseradish peroxidase-conjugated secondary IgG (goat anti-rabbit, cat. no. A16096; 1:10,000; Thermo Fisher Scientific, Inc.; goat anti-mouse, cat. 1706516; 1:10,000; Bio-rad Laboratories, Inc., Hercules, CA, USA) in TBST with 5\% non-fat milk (goat anti-rabbit IgG and goat anti-mouse IgG was purchased from Invitrogen; Thermo Fisher Scientific, Inc.) for $1 \mathrm{~h}$ at room temperature; and then washed a further three times with TBST. Following this, immunoblotting signals were visualized using an ECL chemiluminescence substrate reagent kit (Bio-rad Laboratories, Inc.), and band densities were quantified using Quantity One software 3.0 (Bio-Rad Laboratories, Inc.). CQ was obtained from Sigma-Aldrich; Merck KGaA.

Statistical analysis. Statistical analysis was performed using SPSS version 16.0 for Windows (SPSS, Inc., Chicago, IL, USA). Differences between groups were determined using the Student's t-test or one-way analysis of variance, three or more groups compared using ANOVA. The Scheffe test was used as the post-hoc test. All data are presented as the mean \pm standard deviation. All assays were independently performed a minimum of three times. $\mathrm{P}<0.05$ was considered to indicate a statistically significant difference.

\section{Results}

ITP plasma exhibits decreased rates of apoptosis. In patients with ITP, the presence of apoptotic abnormalities 
A

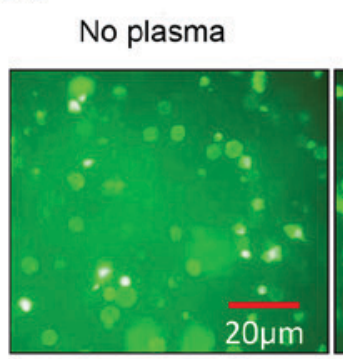
Normal plasma
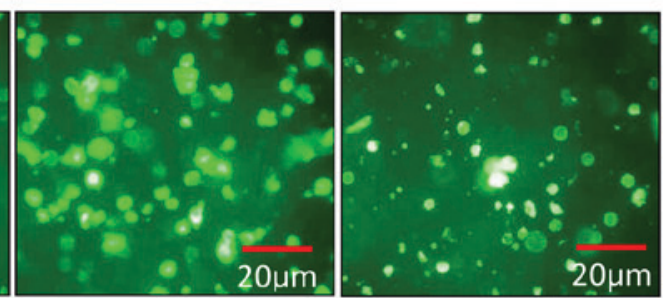

B
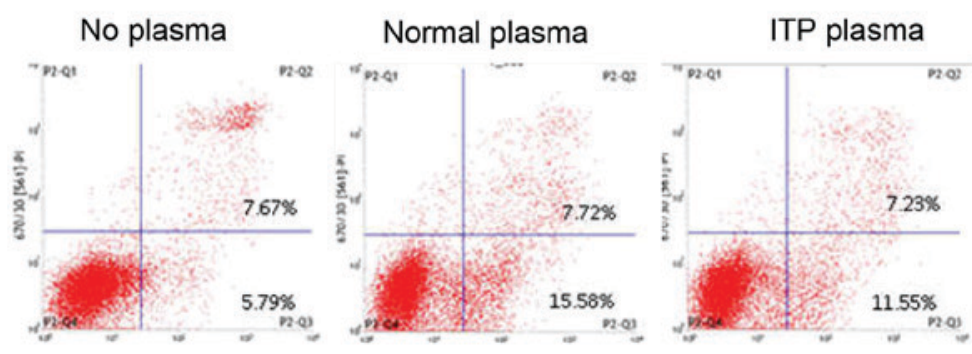
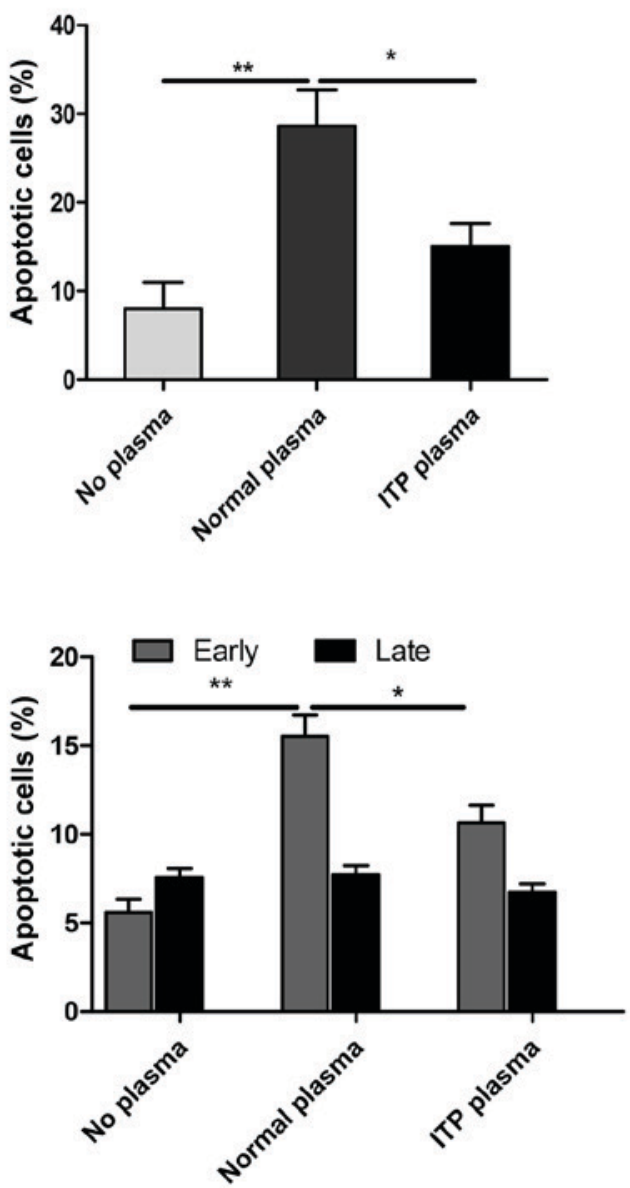

Figure 1. Apoptosis of megakaryocytes is abnormal in ITP plasma group. (A) Apoptosis of megakaryocytes was observed using a fluorescence microscope. (B) Analysis of megakaryocyte apoptosis by Annexin V-FITC/PI flow cytometry. The rate of early apoptosis in the ITP plasma group was decreased compared with normal plasma group, but still increased compared with no plasma group. ${ }^{*} \mathrm{P}<0.05$ and ${ }^{* *} \mathrm{P}<0.01$. ITP, immune thrombocytopenia.

in megakaryocytes is the current diagnostic marker. It is also accepted that megakaryocyte apoptosis in ITP patients is dependent on caspase-3 activation and Bcl-2 (12). To investigate this theory, MEG-01 cells were treated with RPMI-1640 media, 10\% normal plasma, or 10\% ITP plasma and apoptosis was evaluated by flow cytometric analysis of Annexin V-FITC/PI staining. The results demonstrated that the ITP plasma group exhibited decreased levels of apoptosis compared with the normal plasma group (Fig. 1A). The rate of early-stage apoptosis in the ITP plasma group was decreased compared with normal plasma group, but still increased compared with no plasma group (Fig. 1B). Additionally, Fig. 2 revealed that cleaved caspase- 3 and Bax were downregulated in the ITP plasma group compared with the normal plasma group; however, Bcl-2 was upregulated in the ITP plasma group compared with the normal plasma group (Fig. 2). These results confirmed that apoptosis abnormalities exist in ITP megakaryocytes.

Under normal conditions, the changes associated with megakaryocyte differentiation occur mainly in the cytoplasm, with few changes in the nucleus; however, initiation of nuclear lysis was observed in the megakaryocytes. In the ITP plasma group, vacuolization and nuclear condensation were observed in MEG-01 cells compared with the no plasma group; however, there were no such morphological changes in the normal plasma group (Fig. 3).
ITP plasma can induce autophagy. The causes of abnormal apoptosis in the megakaryocytes in ITP patients remain unclear. A previous study have demonstrated that apoptosis is closely associated with autophagy, with apoptosis inhibiting autophagy, or vice versa (13). Therefore, the occurrence of autophagy in megakaryocytes of ITP patients was investigated by Lyso-Tracker Red and ansylcadaverine analysis of MEG-01 cells as an in vitro model. It was demonstrated that the number of Lysosomes in ITP plasma group was increased compared with the normal plasma group (Fig. 4A), indirectly indicating that the proportion of autophagy in the ITP plasma group was higher than that in the normal plasma group, but still lower than that in the no plasma group (Fig. 4B). The western blot also confirmed this result, revealing that ITP plasma exhibited increased levels of Beclin-1 compared with the normal plasma group (Fig. 4C).

ITP plasma can induces autophagy and suppresses apoptosis, which inhibited by CQ. CQ was used as an inhibitor of autophagy to investigate whether ITP plasma suppresses apoptosis by autophagy in MEG-01 cells. Western blotting indicated that when cells were treated with CQ, the ITP plasma was unable to upregulate the apoptosis-related inhibitory proteins (Fig. 5). Flow cytometry demonstrated consistent results with western blotting, in that the ITP plasma induces autophagy otherwise inhibited by CQ (Fig. 6A), which thereby 
A
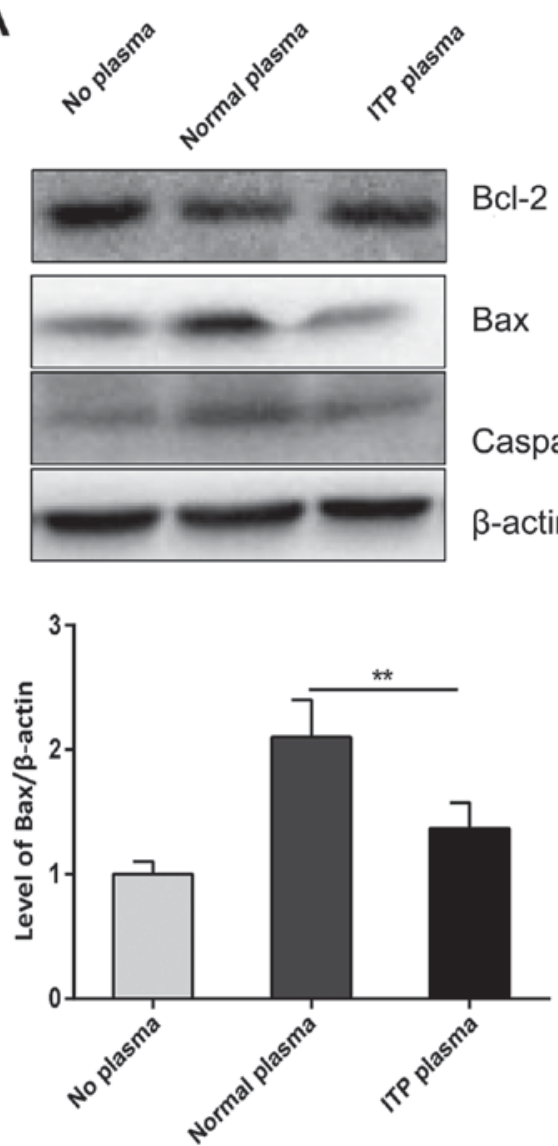

B
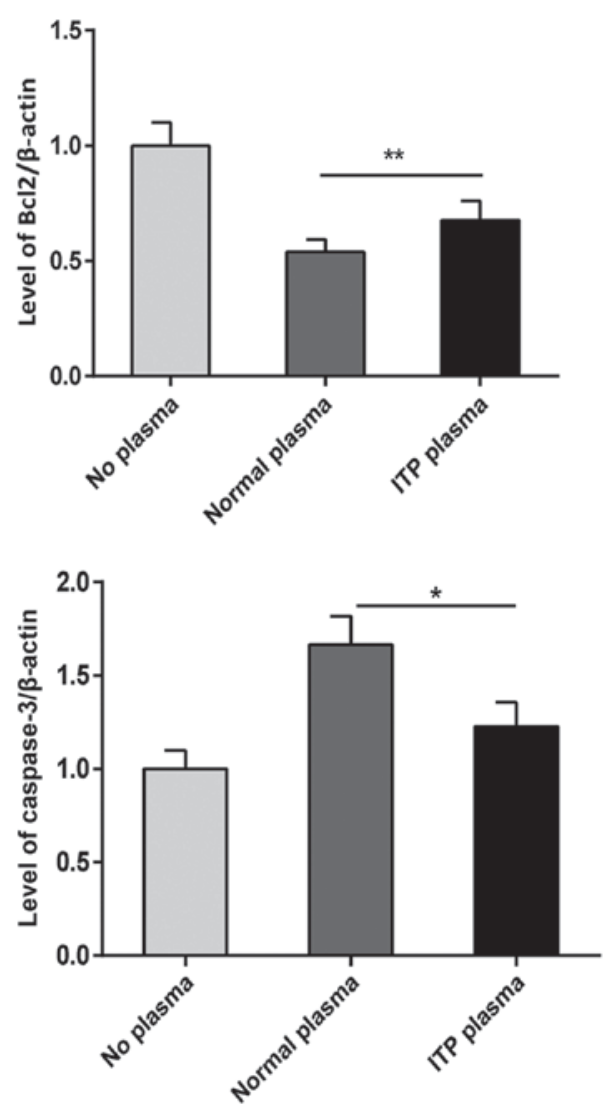

Figure 2. Apoptosis-associated proteins are abnormal in ITP plasma group. (A) Representative blots and (B) quantification of the expression of cleaved Caspase-3, Bcl2 and Bax, as analyzed by western blotting. "P<0.05 and ${ }^{* *} \mathrm{P}<0.01$. Bcl2, B-cell lymphoma-2; Bax, Bcl2-associated X protein; ITP, immune thrombocytopenia.
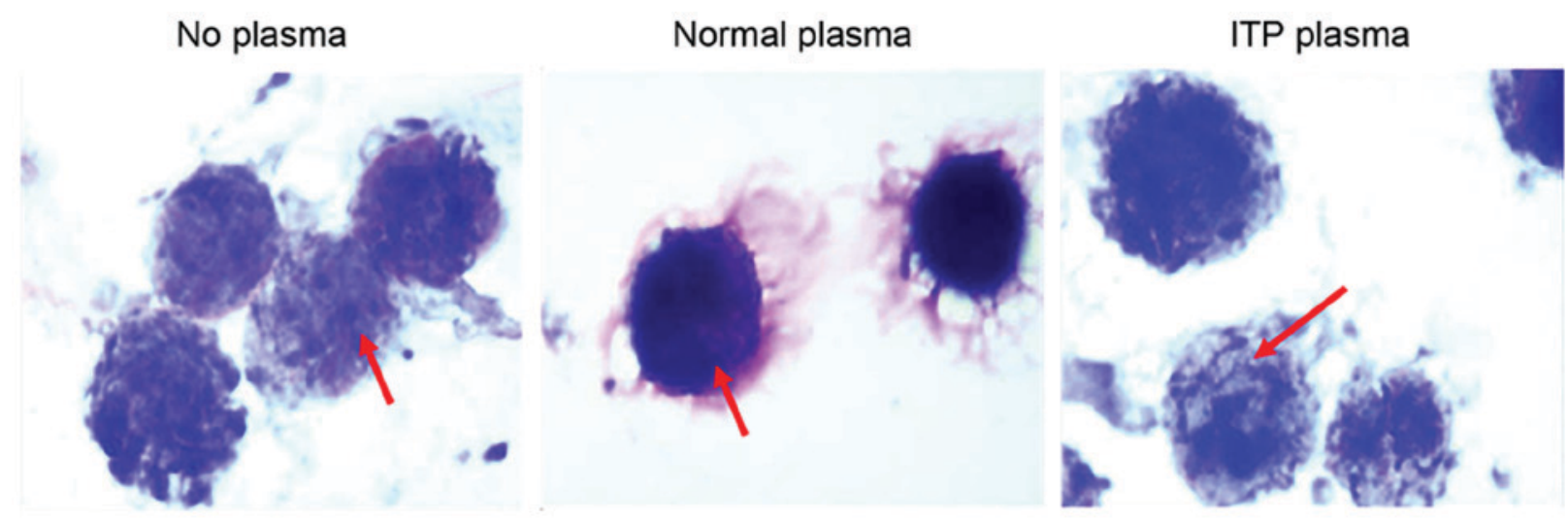

Figure 3. Morphological changes of MEG-01 cells after treatment as described previously. ITP, immune thrombocytopenia.

suppresses apoptosis compared with CQ group (Fig. 6B). Therefore, ITP plasma may serve a key role in development of megakaryocyte autophagy.

\section{Discussion}

Investigations regarding the mechanism of autoimmune diseases have focused mainly on the destruction of platelets, decrease in platelet production and $\mathrm{T}$ lymphocyte immune imbalance $(3,14)$. Studies of the survival of megakaryocytes in ITP patients are rare and few studies have investigated the role of autophagy in ITP. Fabre et al (15) revealed that many autophagic vacuoles are present in the cytoplasm prior to apoptosis, detected via transmission electron microscopy. Houwerzijl et al (10) demonstrated that patients with ITP exhibited mitochondrial and endoplasmic reticulum formation in megakaryocytes, increased vacuolization in the cytoplasm, thickening of the plasma membrane and chromatin condensation in the nucleus; which may be due to para-apoptosis or autophagic cell death. Platelet production depends on compartmentalized caspase activation within megakaryocytes, especially the activation of caspase-3, 
A

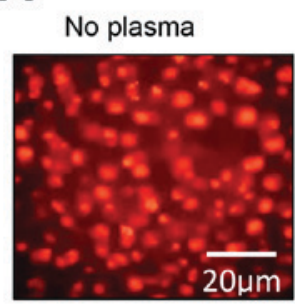

Normal plasma

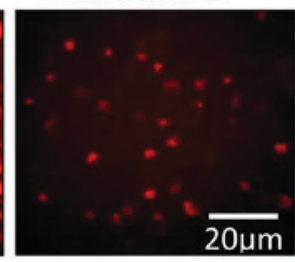

B
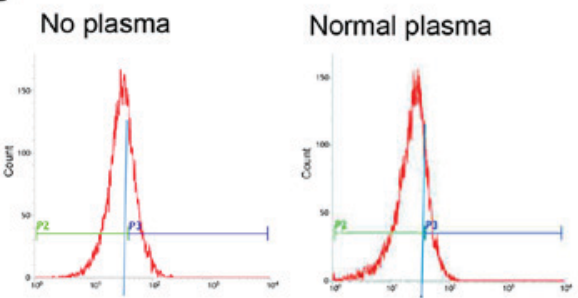

C

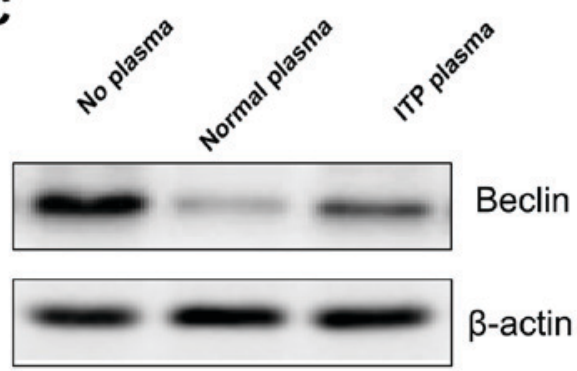

ITP plasma

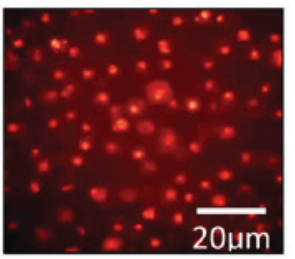

ITP plasma

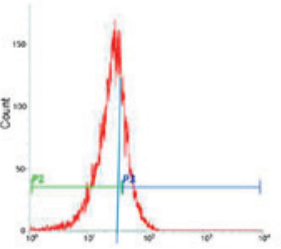

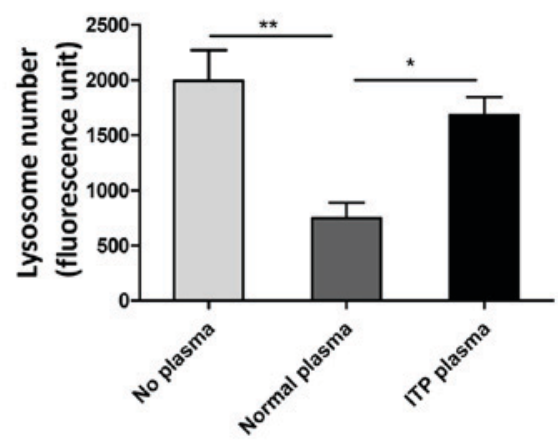

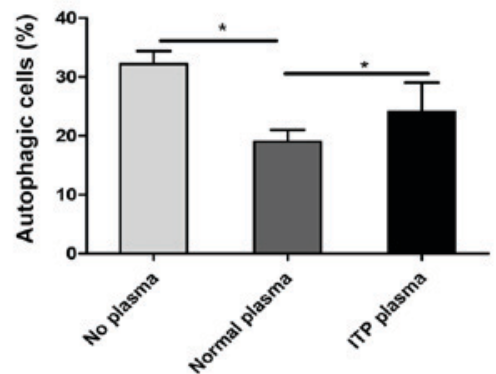

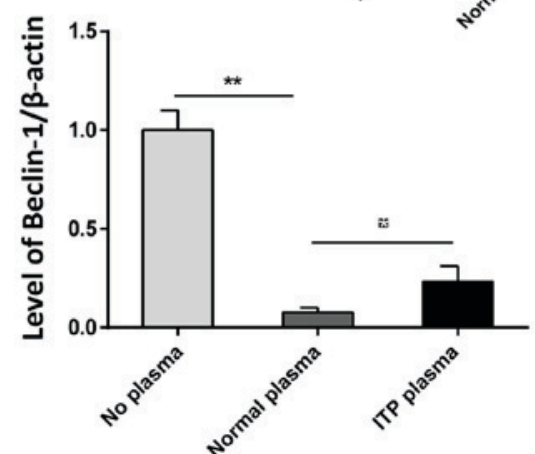

Figure 4. ITP plasma induces autophagy. (A) MEG-01 cell autophagy following treatment described previously. (B) Evaluation of cell autophagy by MDC assay and flow cytometry. (C) ITP plasma can induce the expression of Beclin1, as detected by western blotting. ${ }^{*} \mathrm{P}<0.05$ and ${ }^{* *} \mathrm{P}<0.01$. ITP, immune thrombocytopenia.

A
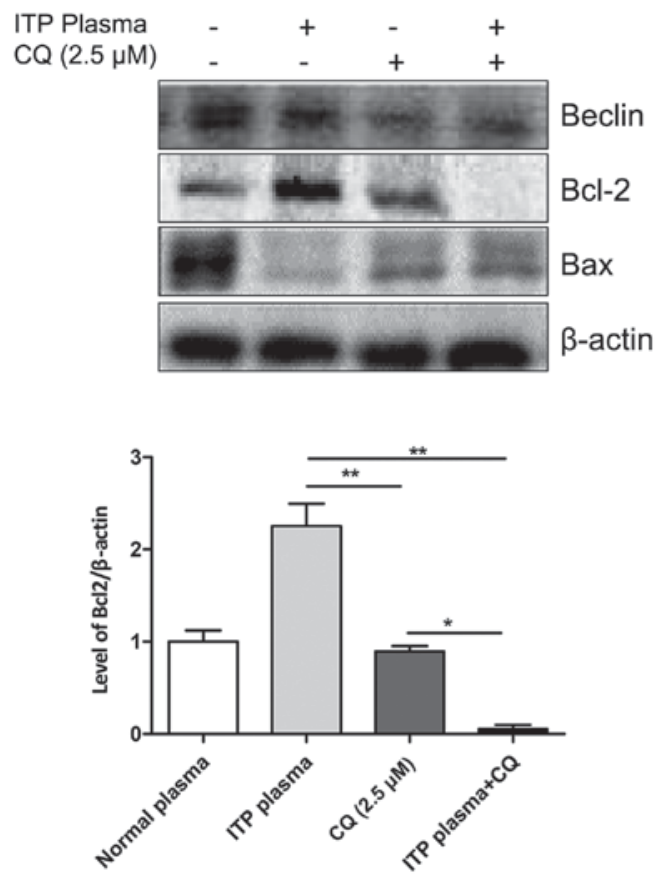

B
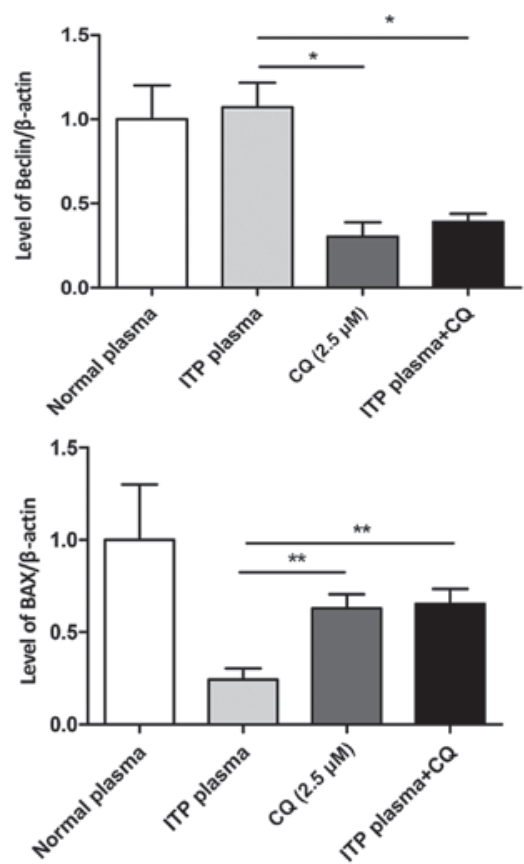

Figure 5. CQ blocks autophagy induced by ITP plasma. (A) CQ suppresses Beclin1 and Bax expression and induces Bcl2 expression. The ITP plasma can induce autophagy proteins inhibited by $\mathrm{CQ}$, thereby suppressing apoptosis relative to the $\mathrm{CQ}$ group. (B) quantification of their expression. ${ }^{*} \mathrm{P}<0.05$ and ${ }^{* *} \mathrm{P}<0.01$. Bcl2, B-cell lymphoma-2; Bax, Bcl2-associated X protein; CQ, Chloroquine; ITP, immune thrombocytopenia. 
A

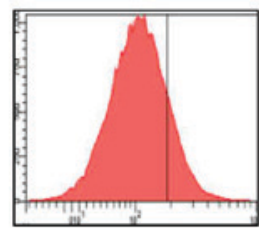

Normal plasma

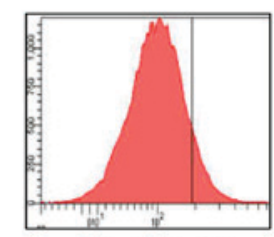

$\mathrm{CQ}(2.5 \mu \mathrm{M})$

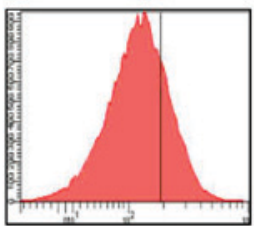

ITP Plasma

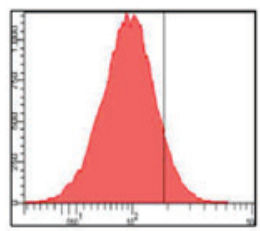

ITP plasma+CQ

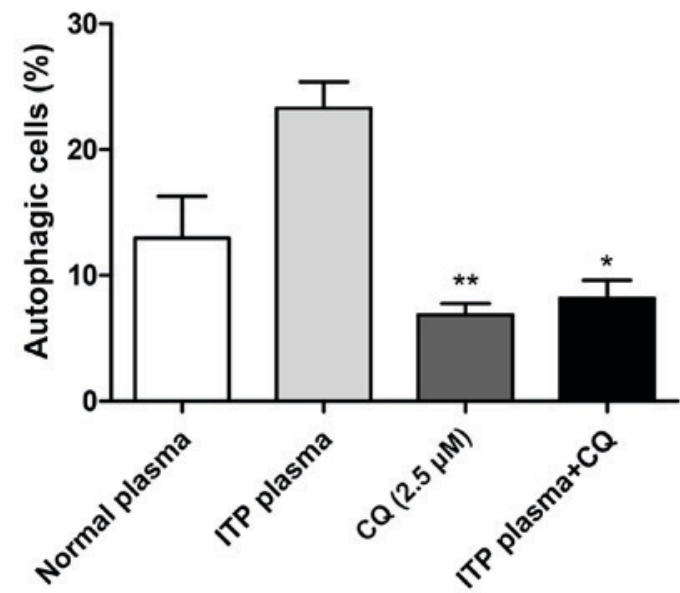

B

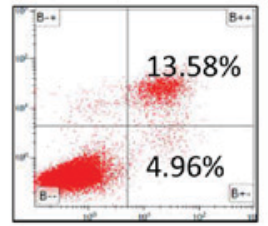

Normal plasma

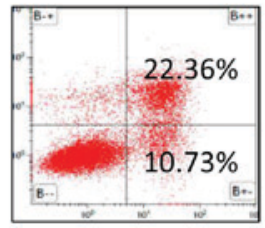

$\mathrm{CQ}(2.5 \mu \mathrm{M})$

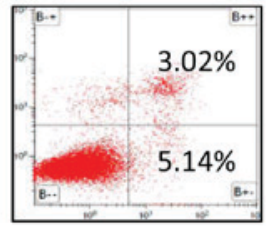

ITP Plasma

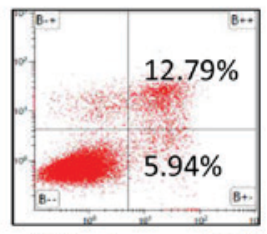

ITP plasma+CQ

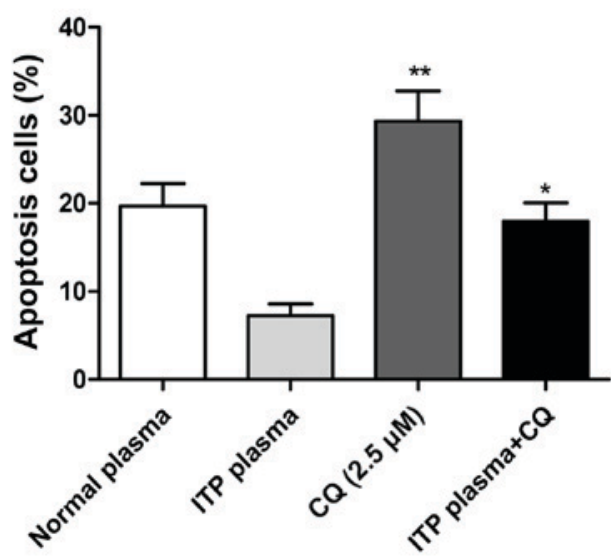

Figure 6. CQ blocks autophagy induced by ITP plasma, as detected by flow cytometry. (A) CQ blocks the ITP plasma induction of autophagy. (B) CQ reverses ITP plasma inhibition of apoptosis. ${ }^{*} \mathrm{P}<0.05$ and ${ }^{* *} \mathrm{P}<0.01$ vs. ITP plasma. $\mathrm{CQ}$, Chloroquine; ITP, immune thrombocytopenia.

which leads to apoptosis (16). The present results indicated that caspase-3 activity decreased in the ITP plasma group, which provides evidence of the apoptotic abnormalities of megakaryocytes in ITP patients. Subsequent investigations of morphological changes and Lyso-Tracker Red/MDC assays confirmed the existence of autophagy in the ITP plasma group. These findings indicated that caspase activation is responsible for autophagy in megakaryocytes of ITP patients, thus affecting the apoptotic process. The apoptotic pathways of megakaryocytes are intrinsic and extrinsic, and both require Bcl-2 involvement (17). Furthermore, recent studies have reported that $\mathrm{Bcl}-2$ is a cross-over point between autophagy and apoptosis in autoimmune disease, tumor, or injury (7,18-20). Furthermore, numerous studies have demonstrated the involvement of $\mathrm{Bcl}-2$ in both apoptosis and autophagy in diverse diseases $(13,21)$. This involvement depends on the formation of complexes containing Bcl-2, Bax and Beclin-1 and their interactions, which determine whether cells enter apoptosis or autophagy. Under conditions of cellular stress, the reduction in Bcl-2 results in a decrease in binding to Beclin-1, leading to an increased free Beclin-1, which promotes autophagy. Alternatively, phosphorylation of Beclin-1 increases Beclin-1-Bcl-2 complexes, displacing Bax from Bcl-2 and resulting in apoptosis (22). The existence of such a change in ITP patients was investigated by evaluation of alterations in cell morphology, apoptosis, autophagy, and protein expression. The present results demonstrated that
Bax and Beclin-1 were downregulated, whereas Bcl-2 was upregulated in ITP plasma compared with normal plasma, and this change may be associated with ER stress; however, this requires further investigation (23). When CQ was used as an inhibitor of autophagy, this inhibited autophagy induced by ITP. However, there are several limitations in the present study. First, more case and functional verification assays in the future studies are required. Furthermore, larger population-based studies are needed in order to confirm the present results.

In conclusion, the present study demonstrated that the ITP plasma induces autophagy and suppresses apoptosis. Inhibition of autophagy may be a novel treatment strategy in ITP, however this requires further investigation.

\section{Acknowledgements}

Not applicable.

\section{Funding}

No funding was received.

\section{Availability of data and materials}

All data generated or analysed during this study are included in this published article. 


\section{Authors' contributions}

TM conceived and designed the present study. ZL performed the experiments and TM performed data analysis and wrote the manuscript.

\section{Ethics approval and consent to participate}

The present study was approved by the Research Ethics Board of the Yongchuan Hospital of Chongqing Medical University and informed consent was obtained from all participants.

\section{Patient consent for publication}

Informed consent was obtained from all participants.

\section{Competing interests}

The authors declare that they have no competing interests.

\section{References}

1. Li J, van der Wal DE, Zhu G, Xu M, Yougbare I, Ma L, Vadasz B, Carrim N, Grozovsky R, Ruan M, et al: Desialylation is a mechanism of Fc-independent platelet clearance and a therapeutic target in immune thrombocy topenia. Nat Commun 6: 7737, 2015

2. Zhou H, Hou Y, Liu X, Qiu J, Feng Q, Wang Y, Zhang X, Min Y, Shao L, Liu X, et al: Low-dose decitabine promotes megakaryocyte maturation and platelet production in healthy controls and immune thrombocytopenia. Thromb Haemost 113: 1021-1034, 2015.

3. Kashiwagi $\mathrm{H}$ and Tomiyama Y: Pathophysiology and management of primary immune thrombocytopenia. Int J Hematol 98 24-33, 2013.

4. Shimizu S, Kanaseki T, Mizushima N, Mizuta $T$, Arakawa-Kobayashi S, Thompson CB and Tsujimoto Y: Role of Bcl-2 family proteins in a non-apoptotic programmed cell death dependent on autophagy genes. Nat Cell Boil 6: 1221-1228, 2004

5. Houwerzijl EJ, Blom NR, van der Want JJ, Esselink MT, Koornstra JJ, Smit JW, Louwes H, Vellenga E and de Wolf JT: Ultrastructural study shows morphologic features of apoptosis and para-apoptosis in megakaryocytes from patients with idiopathic thrombocytopenic purpura. Blood 103: 500-506, 2004.

6. Morishima N and Nakanishi K: Proplatelet formation in megakaryocytes is associated with endoplasmic reticulum stress Genes Cells 21: 798-806, 2016.

7. Lee WS, Sung MS, Lee EG, Yoo HG, Cheon YH, Chae HJ and Yoo WH: A pathogenic role for ER stress-induced autophagy and er chaperone GRP78/BiP in T lymphocyte systemic lupus erythematosus. J Leukoc Biol 97: 425-433, 2015.
8. Yu L, Chen Y and Tooze SA: Autophagy pathway: Cellular and molecular mechanism. Autophagy 14: 207-215, 2018.

9. Liu Q, Luo XY, Jiang H, Yang MH, Yuan GH, Tang Z and Wang $\mathrm{H}$ : Hydroxychloroquine facilitates autophagosome formation but not degradation to suppress the proliferation of cervical cancer SiHa cells. Oncol Lett 7: 1057-1062, 2014.

10. Houwerzijl EJ, Blom NR, van der Want JJ, Vellenga E and de Wolf JT: Megakaryocytic dysfunction in myelodysplastic syndromes and idiopathic thrombocytopenic purpura is in part due to different forms of cell death. Leukemia 20: 1937-1942, 2006.

11. Xue E, Zhang Y, Song B, Xiao J and Shi Z: Effect of autophagy induced by dexamethasone on senescence in chondrocytes. Mol Med Rep 14: 3037-3044, 2016.

12. Yang L, Wang L, Zhao CH, Zhu XJ, Hou Y, Jun P and Hou M: Contributions of TRAIL-mediated megakaryocyte apoptosis to impaired megakaryocyte and platelet production in immune thrombocytopenia. Blood 116: 4307-4316, 2010.

13. Mariño G, Niso-Santano M, Baehrecke EH and Kroemer G: Self-consumption: The interplay of autophagy and apoptosis. Nat Rev Mol Cell Biol 15: 81-94, 2014.

14. Zhou J, Zhou Y, Wen J, Sun X and Zhang X: Circulating myeloid-derived suppressor cells predict disease activity and treatment response in patients with immune thrombocytopenia. Braz J Med Biol Res 50: e5637, 2017.

15. Fabre C, Carvalho G, Tasdemir E, Braun T, Adès L, Grosjean J, Boehrer S, Métivier D, Souquère S, Pierron G, et al: NF-kappaB inhibition sensitizes to starvation-induced cell death in high-risk myelodysplastic syndrome and acute myeloid leukemia. Oncogene 26: 4071-4083, 2007.

16. De Botton S, Sabri S, Daugas E, Zermati Y, Guidotti JE, Hermine O, Kroemer G, Vainchenker W and Debili N: Platelet formation is the consequence of caspase activation within megakaryocytes. Blood 100: 1310-1317, 2002.

17. Kile BT: The role of apoptosis in megakaryocytes and platelets. Br J Haematol 165: 217-226, 2014.

18. Mukhopadhyay S, Panda PK, Sinha N, Das DN and Bhutia SK: Autophagy and apoptosis: Where do they meet? Apoptosis 19: 555-566, 2014.

19. Ruddy SC, Lau R, Cabrita MA, McGregor C, McKay BC, Murphy LC, Wright JS, Durst T and Pratt MA: Preferential estrogen receptor $\beta$ ligands reduce $\mathrm{Bcl}-2$ expression in hormone-resistant breast cancer cells to increase autophagy. Mol Cancer Ther 13: 1882-1893, 2014.

20. Song DD, Zhang TT, Chen JL, Xia YF, Qin ZH, Waeber C and Sheng R: Sphingosine kinase 2 activates autophagy and protects neurons against ischemic injury through interaction with $\mathrm{Bcl}-2$ via its putative BH3 domain. Cell Death Dis 8: e2912, 2017.

21. Su M, Mei Y and Sinha S: Role of the crosstalk between autophagy and apoptosis in cancer. J Oncol 2013: 10271035, 2013.

22. Li M, Gao P and Zhang J: Crosstalk between autophagy and apoptosis: Potential and emerging therapeutic targets for cardiac diseases. Int J Mol Sci 17: 332, 2016.

23. Zhong JT, Xu Y, Yi HW, Su J, Yu HM, Xiang XY, Li XN, Zhang ZC and Sun LK: The BH3 mimetic S1 induces autophagy through ER stress and disruption of Bcl-2/Beclin 1 interaction in human glioma U251 cells. Cancer Lett 323: 180-187, 2012. 